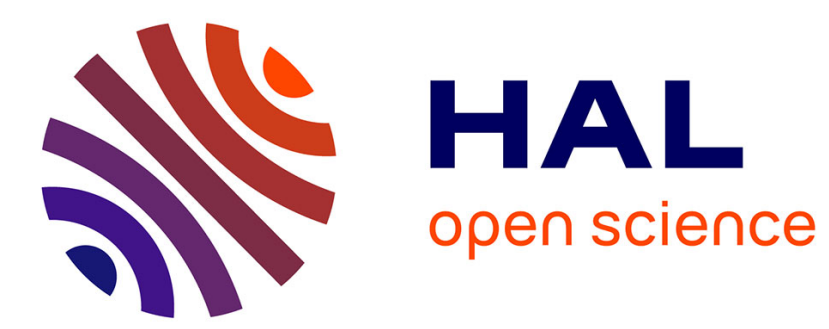

\title{
FORMATION STELLAIRE. ASPECTS THÉORIQUES ET OBSERVATIONNELS
}

\author{
N. Epchtein, Pierre Morel
}

\section{To cite this version:}

N. Epchtein, Pierre Morel. FORMATION STELLAIRE. ASPECTS THÉORIQUES ET OBSERVATIONNELS. Journal de Physique Colloques, 1978, 39 (C1), pp.C1-140-C1-151. 10.1051/jphyscol:1978125 . jpa-00217321

\section{HAL Id: jpa-00217321 https://hal.science/jpa-00217321}

Submitted on 1 Jan 1978

HAL is a multi-disciplinary open access archive for the deposit and dissemination of scientific research documents, whether they are published or not. The documents may come from teaching and research institutions in France or abroad, or from public or private research centers.
L'archive ouverte pluridisciplinaire HAL, est destinée au dépôt et à la diffusion de documents scientifiques de niveau recherche, publiés ou non, émanant des établissements d'enseignement et de recherche français ou étrangers, des laboratoires publics ou privés. 


\title{
FORMATION STELLAIRE. ASPECTS THÉORIQUES ET OBSERVATIONNELS
}

\author{
N. EPCHTEIN
}

Observatoire de Meudon, 92190 Meudon, France

et P. J. MOREL

Observatoire de Nice, Parc Valrose, 06100 Nice, France

\begin{abstract}
Résumé. - Depuis une décennie les progrès technologiques ont permis d'améliorer de façon significative les connaissances observationnelles concernant les régions de formation stellaire. La théorie ne s'est développée qu'avec l'apparition des ordinateurs. L'accord entre théorie et observations est encore loin d'être réalisé.

Dans la première partie on décrit l'instabilité gravitationnelle, puis un processus de fragmentation des nuages denses et enfin les modèles sphériques de protoétoiles. Dans la seconde partie on trouve une description des différents types d'objets observés dans les régions de formation stellaire, puis on expose un modèle d'une des régions les mieux étudiées : Orion.
\end{abstract}

\begin{abstract}
In the recent years, the appearence of new techniques (I. R. and radiointerferometry) have allowed the observation of the site of star formation. In the mean time the new generation of computers have made possible hydrodynamical computations relevant of this problem. But the agreement between theory and observations is still far from now.

In the first part the gravitational instability, some possible process of fragmentation in dense clouds and the contraction of spherical clouds to protostars are described. Then, a review of the different kind of objects observed in regions of star formation is given and Orion, the closest, i. e. the best known, region of star formation is described.
\end{abstract}

1. Introduction générale. - Cet exposé a été préparé avec l'aide d'observateurs et de théoriciens regroupés au sein de la RCP 308.

Etant donné l'étendue du sujet, il nous a semblé préférable de n'en présenter que les aspects les plus marquants.

Ainsi, tant du point de vue observationnel que théorique, on a admis, a priori, que la formation stellaire procède de la condensation des nuages denses ce qui exclut, en particulier, la coalescence des globules de McCrea et la formation dans le noyau galactique d'Ambartzumian (McNally, 1971) [1].

L'évolution du sujet est récente : les théoriciens n'ont été capables de construire des modèles que lorsqu'ils ont eu accès à des calculateurs puissants et ce n'est que depuis la mise au point des techniques d'observation infra-rouge et radio (accès au domaine millimétrique, interférométrique à longue base) que les observateurs ont été capables de s'intéresser efficacement à l'étude des régions de formation stellaire.

Il n'est donc pas surprenant que, pour ces phases initiales de l'évolution stellaire, l'accord entre les résultats des observations et les prévisions théoriques soit encore médiocre. En fait, le scénario imaginé par les théoriciens est, grossièrement, confirmé par les observateurs (Strom et al., 1975) [2], mais il n'est pas encore possible d'interpréter toutes les observations à partir de la théorie. Par exemple on ne sait pas expliquer correctement le fait observationnel de la formation stellaire en groupes situés principalement à la périphérie des nuages.

C'est pour de telles raisons que l'exposé a été séparé en deux parties : théorie et observations, et c'est volontairement qu'on a laissé se dégager l'impression qu'observateurs et théoriciens semblaient parfois traiter des problèmes différents.

Terminons cette introduction générale en donnant un aperçu des difficultés auxquelles se heurtent observateurs et théoriciens.

En Astrophysique il n'est pas possible de faire varier les conditions d'une expérience, et de plus, pour le sujet qui nous retient ici, le voisinage du Soleil n'est pas une région de formation stellaire. Une des plus proches régions est la nébuleuse d'Orion qui se trouve à $500 \mathrm{pc}\left(1,5 \times 10^{19} \mathrm{~m}\right)$. Avec l'instrumentation dont on dispose actuellement, dans les meilleures conditions possibles, les plus fins détails de morphologie que l'on peut espérer y distinguer sont de $2^{\prime \prime}$ en optique et de $0,01^{\prime \prime}$ en interférométrie radio avec une très grande base, soit de 5 à 1000 fois la distance Soleil-Terre $\left(7,5 \times 10^{11}\right.$ à $1,5 \times 10^{14} \mathrm{~m}$ ) ou encore de $10^{3}$ à $2 \times 10^{5} R_{\odot}$. Les observateurs se trouvent aussi confrontés à une 
difficulté d'identification : il n'existe pas de critère observationnel permettant de distinguer si une étoile est rougie par une enveloppe ou par un nuage situé sur la ligne de visée, et la seule donnée dynamique à laquelle on a quelque chance d'accéder est la projection de la vitesse sur la ligne de visée. Le théoricien, lui, semble être plus favorisé : il s'efforce de construire un modèle. Il sait à peu près d'où il part : le nuage dense, et où il doit arriver : le Soleil; mais entre ces deux étapes il n'a pratiquement pas de support observationnel lui permettant de corriger son tir. En plus, dans son modèle, il ne peut évidemment pas mettre toute la physique connue, et en raison de la nature non linéaire des équations des processus physiques négligés au début de l'évolution peuvent devenir prédominants par la suite.

Pour terminer cette introduction sur une note optimiste disons que la confrontation entre observations et observables déduites des modèles théoriques est sur le point de s'amorcer.

2. Aspects théoriques. - 2.1 INTRODUCTION. Une étoile évolue d'autant plus rapidement qu'elle est plus massive. Ainsi une étoile de $3 \mathrm{M}_{\odot}\left(^{1}\right)$ Sirius par exemple, évoluera en quelques $10^{8}$ ans alors que le Soleil aura besoin de trente fois plus de temps. Les étoiles massives $\left(50 \mathrm{M}_{\odot}\right)$ ont une durée de vie très courte par rapport à celle du Soleil si bien que celles qui sont observées actuellement ont été récemment formées. On peut donc admettre, qu'à l'échelle de la Galaxie, ıa formation stellaire est un phénomène continu.

Les observations ont mis en évidence que ces objets jeunes ne sont pas répartis au hasard dans la Galaxie (et les galaxies), mais plutôt qu'ils sont localisés dans des zones bien délimitées (Orion par exemple) où ils sont associés avec des nuages denses : tout ceci va dans le sens de la suggestion de Kant qui, en 1755, proposait déjà une théorie de la formation stellaire à partir de la condensation des nuages interstellaires.

Le premier obstacle auquel on se heurte est celui du moment angulaire. Si l'on suppose qu'un nuage dense standard, de $1 \mathrm{M}_{\odot}$, tournant à la vitesse de la Galaxie, se condense jusqu'aux dimensions du Soleil, on obtient un astre dont la vitesse équatoriale est voisine de celle de la lumière ! Jusqu'à présent aucun processus vraiment satisfaisant n'a permis de perdre ce moment angulaire par exemple en le transférant dans les mouvements orbitaux de systèmes planétaires ou d'étoiles doubles. De même l'influence du champ magnétique est mal comprise. Dans la suite de l'exposé nous ignorerons ces deux difficultés.

Intuitivement on comprend que les processus physiques qui déterminent l'évolution du nuage dense $\left(T \approx 10 \mathrm{~K}, \rho \approx 10^{-19} \mathrm{~g} \cdot \mathrm{cm}^{-3} \approx 10^{5} \mathrm{~cm}^{-3}\right)$ ne sont pas les mêmes que dans une étoile $\left(T \approx 10^{6} \mathrm{~K}\right.$, $\rho \approx 1 \mathrm{~g} \cdot \mathrm{cm}^{-3} \approx 10^{24} \mathrm{~cm}^{-3}$ ). On est donc conduit

(1) $1 M_{\odot}=2 \times 10^{30} \mathrm{~kg}$ à découper l'évolution en un certain nombre d'étapes, susceptibles d'être traitées séparément : les conditions finales de l'une servant de conditions initiales à la suivante, les morceaux se raccordant plus ou moins heureusement. Certaines de ces étapes ont reçu un ample développement, pour d'autres ce n'est que très embryonnaire. Succinctement le scénario peut être décrit de la façon suivante : après sa formation le nuage interstellaire dense est comprimé jusqu'à ce que les conditions de fragmentation soient réalisées, puis fragmenté ; chacun des fragments est enfin condensé jusqu'à l'équilibre quasi-hydrostatique, condition initiale des modèles d'évolution stellaire classique.

Vraisemblablement, c'est sous les effets combinés de l'instabilité thermique (Stein et al., 1972) [3] et de l'instabilité de Parker (action conjointe de la pression magnétique et de la pression de radiation des rayons cosmiques, voir Parker (1970)) [4] que se produit la transition du milieu interstellaire chaud au milieu dense et froid.

Dans la phase froide, l'instabilité gravitationnelle à laquelle est consacré le paragraphe suivant, joue le rôle fondamental. Elle peut être initialisée par une compression. Par exemple, le passage dans une onde de densité (Wielen, 1974) [5], expliquerait la présence d'un grand nombre d'étoiles jeunes au voisinage des bras spiraux.

2.2 L'INSTABILITÉ GRAVITATIONNELLE. - Un nuage de gaz parfait, sphérique, isotherme, homogène s'effondrera gravitationnellement si les forces de gravitation qui tendent à lier les molécules entre elles sont supérieures aux forces d'agitation thermique qui tendent à les éloigner. Sous la forme d'un bilan d'énergie on doit donc avoir :

| énergie gravitationnelle | > énergie thermique |

$$
\frac{3}{5} \frac{G M^{2}}{r} \quad>\quad \frac{3}{2} M \frac{R T}{\mu}
$$

où $G$ est la constante de la gravitation et $R$ celle des gaz parfaits, quant à $M, r, T, \mu$, ils désignent respectivement la masse, le rayon, la température et la masse moléculaire moyenne du nuage. $\rho$ étant la densité, il y aura instabilité si $M$ est supérieur à une valeur critique $M_{\mathrm{c}}\left({ }^{2}\right)$ :

$$
M_{\mathrm{c}}=4,24 \times 10^{-11} \mu^{-3 / 2} T^{3 / 2} \rho^{-1 / 2} \mathrm{M}_{\odot} .
$$

C'est le critère de Jeans.

Il faut souligner que ce critère ne donne qu'une condition suffisante pour que l'instabilité gravitationnelle se produise, et qu'il ne prétend pas décrire l'effondrement en lui-même, puisqu'il néglige les effets dynamiques et le transfert du rayonnement qui jouent un rôle primordial au cours de l'évolution ultérieure.

(2) Avec des formulations plus raffinées du critère (théorème du viriel, pression extérieure, ...) le coefficient numérique peut, suivant le cas, varier de $3 \times 10^{-11}$ à $6 \times 10^{-11}$. 
Ainsi, un nuage de $60 \mathrm{M}_{\odot}$ à la limite de l'instabilité de Jeans s'effondrera, mais l'objet résultant sera une étoile de $17 \mathrm{M}_{\odot}$ : plus de $70 \%$ de la masse initiale ayant été rejetée par la pression de radiation (Appenzeller et Tscharnuter, 1974) [6]. La figure 1 montre que les conditions de l'instabilité gravitationnelle peuvent se trouver réunies dans un nuage dense $\left(M_{\mathrm{c}}>1\right.$ à $\left.10^{2} \mathrm{M}_{\odot}\right)$ à la rigueur dans le milieu neutre $\left(M_{\mathrm{c}}>10^{3}\right.$ à $\left.10^{5} \mathrm{M}_{\odot}\right)$ mais certainement pas dans le milieu chaud et dilué car la masse critique est supérieure à celle des nuages observés. Les masses typiques

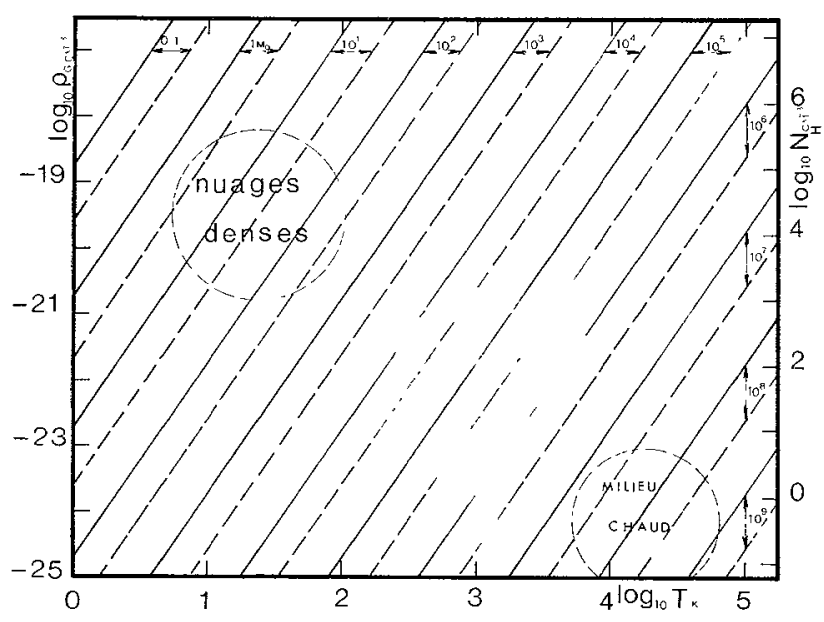

FIg. 1. - Critère de Jeans :

$$
\log _{10} \rho=-2 \log M_{\mathrm{c}}-20,75+3 \log _{10} T
$$

courbes en trait plein correspondant à $\mu=1$.

$$
\log _{10} \rho=-2 \log M_{\mathrm{e}}-21,33+3 \log _{10} T
$$

courbes en tirets correspondant à $\mu=2$. Dans la phase chaude un nuage n'est pas instable sous l'effet de la gravité car les masses nécessaires sont trop élevées. Les masses instables sont, dans les nuages denses, de 1 à $10^{2} \mathrm{M}_{\odot}$.

des nuages denses étant de l'ordre de $10^{2}$ à $10^{5} \mathrm{M}_{\odot}$ il faut faire appel à un processus de fragmentation pour subdiviser un nuage en entités ayant des masses de l'ordre des masses stellaires.

Dans le critère de Jeans le nuage initial est supposé sphérique, or lorsqu'on regarde un nuage dense, il est difficile de croire en une quelconque symétrie ! On a envie de dire que les contrastes de densité forment des germes de condensation qui fragmentent le nuage. Malheureusement une telle idée n'a pas, jusqu'à présent, mené aux résultats escomptés pour différentes raisons :

1) Ce n'est que dans certaines conditions assez restrictives que les contrastes de densité semblent suffisants pour entraîner une fragmentation (McNally, 1971) [1]. (Il faut toutefois préciser que les calculs ont été effectués avec une seule dimension spatiale.)

2) Au cours de tels processus la turbulence dans le nuage devrait être importante contrairement aux observations qui semblent indiquer que la formation stellaire se produit dans des zones calmes (Reddish, 1975) [7].

Pour un nuage dense la théorie de la fragmentation la plus séduisante est basée sur l'instabilité de pression créée au moment de la formation des molécules $\mathrm{H}_{2}$ mais le modèle n'est encore qu'embryonnaire.

2.3 LA FRAgmentation D'UN NUAGE DENSE. Considérons un nuage dense $\left(\rho \approx 10^{3}-10^{4} \mathrm{~cm}^{-3}\right)$ d'hydrogène atomique en équilibre hydrostatique : les forces de pression équilibrent en tous les points les forces de gravité. Supposons que les conditions physiques soient telles que le taux de formation des molécules $\mathrm{H}_{2}$ soit important $T \approx 10 \mathrm{~K}$. Au moment de la formation d'une molécule $\mathrm{H}_{2}$ par association de deux atomes d'H, la pression locale $(P=n k T)$ se trouve divisée par 2 . Si la chaleur dégagée par la réaction est perdue pour le gaz, l'équilibre hydrostatique est rompu et il y a effondrement. En termes du critère de Jeans, la formation des molécules $\mathrm{H}_{2}$ revient à multiplier $\mu$ par 2, c'est-à-dire à multiplier la masse critique par 0,35 $\left(2^{-3 / 2}\right)$.

On pense que les molécules $\mathrm{H}_{2}$ sont formées par le processus suivant : par adsorption, les grains de poussière interstellaire retiennent les atomes d'H qui les rencontrent au cours des mouvements dûs à l'agitation thermique. Si, sur un grain, les atomes d'H restent collés suffisamment longtemps pour que les rencontres (donc la possibilité d'association) soient nombreuses, le taux de formation des molécules $\mathrm{H}_{2}$ sera important. La chaleur de la réaction est transmise au grain mais, pour des températures supérieures à $\approx 3 \mathrm{~K}$, elle est totalement négligeable dans le bilan énergétique de celui-ci. Pratiquement, si la température du grain est inférieure à une température critique $T_{\mathrm{c}} \approx 20 \mathrm{~K}$ (Reddish, 1975) [7], chaque paire d'atomes $\mathrm{H}$ rencontrant le grain se transforme en une molécule $\mathrm{H}_{2} . T_{\mathrm{c}}$ ne dépend que de la nature et de la forme du grain.

Dans un nuage la température des grains s'ajuste de façon à ce qu'il y ait équilibre entre l'énergie reçue (Rayons cosmiques, U. V., $\gamma, \mathbf{X}, \mathrm{I}$. R., collisions) et l'énergie perdue par le rayonnement. Il s'y produit nécessairement des fluctuations de température dont l'échelle de longueur est l'épaisseur optique. On peut donc admettre que le nuage se trouve découpé en cellules d'épaisseur optique unité ; chacune d'elles ayant une température à peu près constante et se comportant à peu près indépendamment des autres quant au taux de formation des molécules $\mathrm{H}_{2}$.

Dès que la densité de molécule $\mathrm{H}_{2}$ augmente, l'énergie reçue des sources extérieures R. C., U. V., $\gamma, \mathrm{X}$, I. R. ... diminue, entraînant une diminution de la température des grains, par ailleurs les pertes radiatives dues à la molécule $\mathrm{H}_{2}$ augmentent : il y a accélération du processus.

Estimons la masse des fragments : pour une cellule de rayon $r$, si $Q$ est le coefficient d'extinction des grains de rayon $a, n_{\mathrm{g}}$ leur nombre par unité de volume (Reddish, 1975) [7]. 


$$
\tau=1=\Pi a^{2} Q n_{\mathrm{g}} r
$$

la masse de la cellule est :

$$
\begin{aligned}
M=\frac{4}{3} \Pi m_{\mathrm{H}}\left(\Pi a^{2} Q\right)^{-3}\left(\frac{n_{\mathrm{g}}}{n_{\mathrm{H}}}\right)^{-3} n_{\mathrm{H}}^{-2}= \\
=2,8 \times 10^{-29}\left(\frac{n_{\mathrm{g}}}{n_{\mathrm{H}}}\right)^{-3} n_{\mathrm{H}}^{-2} \mathrm{M}_{\odot} .
\end{aligned}
$$

Pour que la cellule devienne un fragment individualisé, il faut que le temps caractéristique de l'effondrement gravitationnel du nuage tout entier soit supérieur au temps caractéristique de transformation du gaz de la cellule d'H en $\mathrm{H}_{2}$.

Pour qu'à son tour le fragment puisse se condenser, il faut qu'il satisfasse la condition de Jeans, si bien que :

$2,2 \times 10^{-15}\left(\frac{n_{\mathrm{g}}}{n_{\mathrm{H}}}\right)^{-5 / 3}>M>6,3 \times 10^{12}\left(\frac{n_{\mathrm{g}}}{n_{\mathrm{H}}}\right)$ en $\mathrm{M}_{\odot}$

soit

$2,5 \times 10^{5} \mathrm{M}_{\odot}>M>6,3 \mathrm{M}_{\odot} \quad\left(n_{\mathrm{g}} / n_{\mathrm{H}}=10^{-12}\right)$.

La limite inférieure laisse à penser que le processus décrit peut permettre d'isoler des fragments de masse stellaire.

Comme l'indiquent les observations, les fragments les plus massifs seront formés à la périphérie du nuage à cause de la dépendance de $M$ en $n_{\mathrm{H}}^{-2}$, (en admettant que le gradient de densité soit dirigé vers le centre) (Fig. 2).

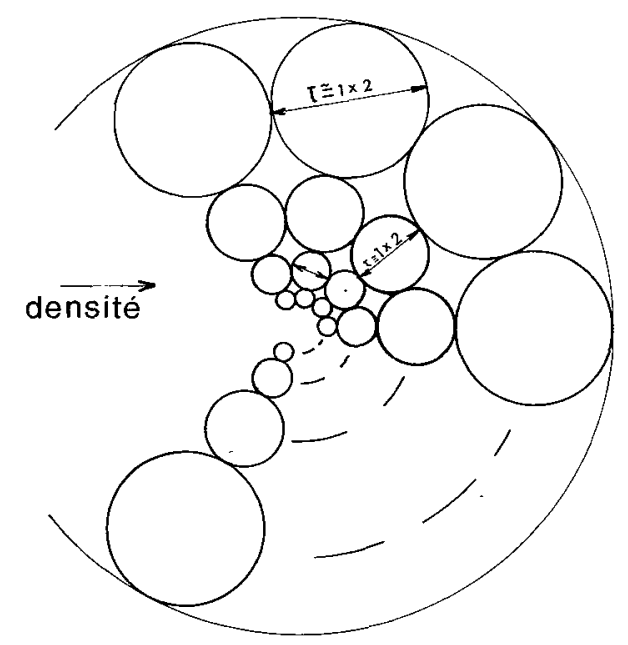

FIG. 2. - Fragmentation d'un nuage dense. Les conditions thermiques dans un globule de rayon optique $\tau \approx 1$ sont à peu près constantes et indépendantes de celles qui règnent dans les autres globules. Les globules seront d'autant plus grands et plus massifs qu'ils sont plus extérieurs.

Aucun calcul n'a encore été fait pour tenter de décrire l'individualisation et l'évolution des fragments (collisions et sub-fragmentations), mais cette théorie, toute qualitative, est la théorie de la fragmentation qui a le plus de support observationnel et qui présente l'avantage de ne dépendre que du paramètre $n_{\mathrm{g}} / n_{\mathrm{H}}$ déductible des observations.

2.4 CONDENSATION DES FRAGMENTS. - On arrive maintenant à la troisième étape : la condensation des fragments. C'est là où le travail quantitatif est le plus important. En supposant que la sphéricité initiale est conservée durant toute la contraction $\left(^{3}\right)$ on peut construire des modèles numériques qui deviennent de plus en plus précis et détaillés.

2.4.1 Conditions initiales. - La théorie de la fragmentation n'étant pas suffisamment quantitative pour proposer une description physique des fragments au début de leur condensation, il est généralement admis que ceux-ci satisfont la condition d'instabilité gravitationnelle. Ainsi la donnée de la température et de la masse totale conduit à la densité et au rayon pour une sphère homogène, isotherme (formule (1)).

La température, la composition chimique, la teneur en poussières ont des valeurs à peu près invariables d'un modèle à l'autre $\left(T \simeq 10 \mathrm{~K}, n_{\mathrm{g}} / n_{\mathrm{H}} \approx 10^{-12}\right)$. On a d'ailleurs montré que des modifications raisonnables de ces paramètres libres n'entraîneraient pas de grandes modifications de l'évolution ultérieure.

Ainsi pour $1 \mathrm{M}_{\odot}$ la densité avoisine $10^{-19} \mathrm{~g} . \mathrm{cm}^{-3}$ et le rayon $10^{17} \mathrm{~cm}$.

2.4.2 Equations. - En utilisant la variable de Lagrange $m$ : masse contenue à l'intérieur d'une sphère de rayon initial $r$ :

$$
m=\int_{0}^{r} 4 \Pi x^{2} \rho(x) \mathrm{d} x
$$

( $\rho=1 / v$ densité), le système des équations à résoudre est le suivant:

$\frac{1}{\rho}=V=4 \Pi r^{2} \frac{\partial r}{\partial m}$ équation de continuité

$\frac{\partial u}{\partial t}+4 \Pi r^{2} \frac{\partial P}{\partial m}+\frac{G m}{r^{2}}=0$ équation du mouvement

$$
\begin{aligned}
& \frac{\partial E}{\partial t}+p \frac{\partial V}{\partial t}+\Lambda=0 \quad \text { équation d'énergie } \\
& u=\frac{\partial r}{\partial t} .
\end{aligned}
$$

C'est le système des équations de l'hydrodynamique écrit en symétrie sphérique. $u(m, t)$ est la vitesse à l'abscisse Lagrangienne $m$ à l'instant $t, P$ est la pression, $E$ l'énergie interne spécifique et $\Lambda$ le bilan pertesgains d'énergie par unité de temps et de masse.

Les termes (du second ordre) dûs à la viscosité sont

(3) On ne peut donc pas tenir compte ni du moment angulaire ni du champ magnétique. 
négligeables dans les conditions présentes. Une équation d'état du type gaz parfait relie $E, P, V$ et $T$.

Dans l'équation du mouvement, c'est le terme $G m / r^{2}$ qui constitue le système des forces extérieures : l'état dynamique sera déterminé par la balance entre forces de gravité et forces de pression.

Quand on supprime le terme de pression, l'équation $\mathrm{du}$ mouvement s'intègre analytiquement et on obtient le temps caractéristique de chute libre

$$
t_{\mathrm{ff}}=\left(\frac{3 \Pi}{32 G \rho}\right)^{1 / 2}
$$

En l'absence des forces de pression, c'est le temps qui serait nécessaire pour rassembler toute la masse au centre.

$t_{\text {ff }}$ variant en $\rho^{-1 / 2}$, le temps de chute libre sera plus petit dans les régions plus concentrées ce qui a pour effet d'accélérer la concentration dans ces régions diminuant d'autant $t_{\mathrm{ff}} \ldots$ d'où la très rapide condensation centrale mise en évidence par les premiers modèles numériques.

2.4.3 Conditions aux limites. - $\mathrm{Au}$ centre $\mathrm{du}$ nuage, $r(0, t)=0$ et $u(0, t)=0$ à cause de l'hypothèse de sphéricité. A l'extérieur $(m==M)$ il faut une condition aux limites. Si l'on admet que les étoiles se forment en groupe, on peut supposer que la frontière initiale du nuage est fixe $(r(M, t)=t(M, 0)$ pour tout $t)$ ce qui revient à poser $U(M, t)=U(M, 0)=0$, ou bien, si l'on accepte qu'une étoile puisse se former isolément, on admettra que le milieu extérieur suit la condensation et alors $P(M, t)=P(M, 0)$. La plupart des modèles furent calculés avec la première hypothèse. Qualitativement, les deux types de conditions aux limites donnent des résultats équivalents.

2.4.4 Codes numériques utilisés. - Dans un tel travail la difficulté est la mise au point du code numérique. C'est le côté ingrat, obscur, mais fondamental. A cause de son aspect rébarbatif on dira seulement que les codes numériques utilisés dans les modèles sont, pour la plupart, implicites et dérivés de celui de Richtmyer et Morton (1967) [8].

2.4.5 Calcul du bilan pertes-gain. - Suivant l'endroit où l'on se trouve dans le nuage et l'état d'évolution, les processus physiques intervenant dans ce bilan seront fort différents : initialement on a un nuage dense où la formation des molécules joue un rôle fondamental (Kolesnik, 1976) [9], au contraire vers la fin de l'état de protoétoile les réactions thermonucléaires s'amorcent au centre et la convection peut jouer un rôle important dans l'évacuation de l'énergie radiative, alors que dans l'enveloppe, pratiquement en chute libre sur le noyau, le transfert de l'énergie radiative sera compliqué par la formation de raies dans un champ de vitesse.

Il est évident que toutes ces difficultés ne peuvent être résolues à la fois. Chronologiquement les modèles ont d'abord eu pour but de résoudre le problème hydro- dynamique, le terme $\Lambda$ a été grossièrement estimé en utilisant l'hypothèse de diffusion pour calculer le flux radiatif, puis on a tenu compte de l'épaisseur optique finie et des effets de sphéricité. Actuellement on cherche à tenir compte plus correctement des discontinuités introduites par les ondes de choc en même temps qu'un transfert assez précis est introduit dans les couches extérieures de façon à pouvoir calculer des observables.

2.4.6 Description de l'évolution. (Modèles unidimensionnels). - 2.4.6.1 Phase de chute libre $\left(\approx 1 t_{\mathrm{ff}} \approx 2 \times 10^{5}\right.$ ans pour $\left.1 \mathrm{M}_{\odot}\right)$. - Dès l'instant initial, le nuage s'effondre. L'énergie thermique libérée par compression n'est pas capable d'assurer le rayonnement thermique de l'objet qui se refroidit jusqu'à des températures très basses. Si l'on suppose que les sources de chauffage du nuage ne sont pas coupées à l'instant initial, l'évolution est d'abord presque isotherme. Dans cette première phase l'énergie gravitationnelle libérée est transformée en énergie cinétique. Au voisinage du centre se développe un fort gradient de densité qui, après environ 1 à $1,5 t_{\mathrm{ff}}$, se transforme en une onde de choc (discontinuité). A l'intérieur du choc se trouve un noyau dense en équilibre quasi-hydrostatique dont l'épaisseur optique est largement supérieure à 1 , L'objet est devenu une protoétoile. Une grande partie de l'énergie cinétique est transformée en énergie interne sur l'onde de choc. L'objet commence alors à rayonner son énergie gravitationnelle. Typiquement pour $1 \mathbf{M}_{\odot}$ le noyau renferme $5 \times 10^{-3} \mathrm{M}_{\odot}$ et son rayon avoisine $10^{3} R_{\odot}$. La température centrale est de l'ordre de $200 \mathrm{~K}$, et la densité de $10^{-8} \mathrm{~g} . \mathrm{cm}^{-3}$ environ, celle-ci a donc été multipliée par un facteur $10^{11}$ (Larson, 1973) [10].

Ce premier noyau, évoluant de façon quasi-hydrostatique, s'échauffe et, lorsque la température atteint $2000 \mathrm{~K}$ environ, la dissociation des molécules $\mathrm{H}_{2}$ rompt l'équilibre de pression, il y a de nouveau effondrement puis formation d'une seconde onde de choc bordant un noyau dense renfermant $10^{-3} \mathrm{M}_{\odot}$ à peu près. C'est ce second noyau central qui deviendra l'étoile finale (l'effondrement et la formation d'un noyau dense ne se reproduisant pas au moment de l'ionisation des atomes d'H).

2.4.6.2 Phase d'accrétion $\left(\approx 2 t_{\mathrm{ff}} \approx 4 \times 10^{5}\right.$ ans pour $\left.1 \mathrm{M}_{\odot}\right)$. - Pendant cette phase, la masse de la partie centrale va augmenter aux dépens de celle de l'enveloppe. Une ou plusieurs zones convectives peuvent se développer dans le noyau mais celles-ci ne contribuent réellement au bilan thermique que lorsque la masse initiale est inférieure à $\approx 3 \mathrm{M}_{\odot}$ (Westbrook et Tarter, 1975) [11].

Pour la suite de l'évolution, suivant la masse du nuage initial, les modèles mettent en évidence une alternative :

(1) $M \lesssim 3 \mathrm{M}_{\odot}\left({ }^{4}\right)$ : la quasi-totalité de l'enveloppe se trouve accrétée dans le noyau lorsque les réactions thermonucléaires se trouvent amorcées : la masse de 
l'étoile est très voisine de celle du nuage dont elle est issue.

(2) $M \gtrsim 3 \mathrm{M}_{\odot}\left({ }^{4}\right):$ une partie seulement de la masse totale se trouve dans le noyau lorsque la pression de radiation due au corps central est capable de repousser l'enveloppe: la protoétoile devient une étoile à cocon. La masse de l'étoile peut être nettement inférieure $(30 \%$ seulement) à celle du nuage initial.

2.4.6.3 Phase finale. - Pour une étoile de masse inférieure à $\approx 3 \mathrm{M}_{\odot}$, lorsque l'enveloppe se dissipe, le noyau central devenant visible il se produit une brusque augmentation de la luminosité (Narita et al., 1970) [12] et, vraisemblablement une modification importante de la nature du spectre observé.

Avec un code numérique construit pour rendre compte des effets hydrodynamiques, il était hasardeux de vouloir décrire ces phases finales. Ce n'est que récemment qu'on a amélioré les algorithmes afin de pouvoir calculer l'évolution temporelle du spectre (Bertout, 1976) [13] ou décrire la formation des cocons (Yorke et Krïgel, 1977) [14].

2.5 LiAISON AVEC LeS OBSERVATIONS. - Il a déjà été dit qu'il était prématuré de prétendre pouvoir identifier des objets stellaires à des modèles théoriques de protoétoiles dans une phase précise de leur évolution. On a quand même recherché à rattacher les faits les plus marquants de la théorie à des observations.

Qualitativement le modèle de la protoétoile entourée d'une enveloppe de poussières rayonnant en $I$. $R$. est confirmé par l'existence de sources I. R. ponctuelles dans les nuages denses. Lorsque le noyau central apparaît, les paramètres accessibles à l'observateur varient avec une petite échelle de temps, en particulier, certains modèles prévoient une rapide augmentation de la luminosité qui a été rapprochée de celle des étoiles à flares (FU Orionis). A la limite $\approx 3 \mathrm{M}_{\odot}$, à la bifurcation entre les deux types d'évolution possibles, vers la fin de l'état de protoétoile, on devrait observer le spectre de l'étoile centrale à travers un résidu d'enveloppe en chute libre, des vitesses de $300 \mathrm{~km} . \mathrm{s}^{-1}$ pourraient être atteintes, valeurs en accord avec de récentes observations d'Appenzeller et Wolf (1977) [15] sur l'étoile S CrA (type T Tau).

2.6 Modèles BI ET TRIDIMENSIONNELS. - Les modèles sphériques sont incapables de tenir compte du moment angulaire et du champ magnétique.

On peut étudier l'influence du moment angulaire dans des modèles en symétrie axiale, ceux-ci ont mis en évidence, sous certaines conditions, la formation d'un anneau qui, instable, se fragmenterait.

Une tentative, très controversée, de modèle à trois dimensions a été entreprise. Les résultats montrent la formation de systèmes stellaires, simples, doubles, triples et quadruples à partir d'un nuage initial.

(4) Cette valeur serait comprise entre 1 et $5 \mathrm{M}_{\odot}$.
A deux et trois dimensions, les difficultés numériques deviennent rapidement très grandes, non seulement en termes de coût et de puissance d'ordinateur mais surtout en termes de codes numériques stables à découvrir.

2.7 CONCLUSIONS SUR L'ASPECT THÉORIQUE. - La plupart des processus permettant au nuage dense de se condenser ont pour effet de l'amener dans les condi tions de l'instabilité gravitationnelle qui semble être la cause fondamentale de la formation stellaire.

La fragmentation en subcondensations de masses stellaires ne sera comprise que lorsqu'on saura construire des modèles à trois dimensions, incluant le moment angulaire, le champ magnétique et les effets dynamiques. Mais s'il est bien clair que seuls ces modèles pourront donner une description satisfaisante de la formation des étoiles, il n'en reste pas moins vrai que l'hypothèse de symétrie sphérique est réalisée dans la nature : il serait paradoxal que le Soleil soit la seule étoile sphérique de l'Univers! Il est donc souhaitable d'améliorer les modèles unidimensionnels, les techniques numériques mises au point, en particulier en ce qui concerne le traitement des discontinuités et du transfert radiatif pourront non seulement s'appliquer aux cas multidimensionnels mais aussi à d'autres branches de l'astrophysique et même de la physique.

3. Aspects observationnels. - -3.1 LES vOIES NOUVELLES DE L'OBSERVATION. - L'étude observationnelle des objets stellaires en formation et des processus physiques de formation stellaire ont progressé de manière décisive depuis que les astronomes ont accès aux domaines spectraux infra-rouge et millimétrique.

On a pu ainsi observer des objets invisibles optiquement soit parce qu'ils sont trop froids $(T<1000 \mathrm{~K})$, soit parce qu'ils sont cachés par de la matière absorbante dans le domaine visible, soit parce qu'ils sont trop diffus ou contiennent des molécules dont les raies sont émises dans le domaine millimétrique ou centimétrique.

On a pu mettre en évidence une grande quantité de matière sous forme :

1) De molécules détectées par leurs transitions de rotation dans les domaines centimétrique et millimétrique. Les plus abondantes (hormis $\mathrm{H}_{2}$ ) sont $\mathrm{CO}$, $\mathrm{CS}, \mathrm{HCN}, \mathrm{H}_{2} \mathrm{CO}, \ldots$ (La molécule $\mathrm{CO}$ est détectable même dans des milieux diffus $\lesssim 10^{2} \mathrm{~cm}^{-3}$ ).

2) De grains de poussière solide (dont on avait déjà montré l'existence par leur absorption sélective optique et par les mesures de polarisation) observés directement par leur rayonnement thermique infra-rouge. On montre ainsi qu'un centième environ de la masse des nuages se trouve sous forme de grains solides.

3) D'étoiles de grande luminosité infra-rouge mais très faibles ou invisibles en optique trouvées associées aux nuages moléculaires et aux régions $\mathrm{H}$ II. 
4) De sources émettant un rayonnement maser dans les raies des molécules $\mathrm{OH}$ et $\mathrm{H}_{2} \mathrm{O}$ également découvertes à proximité de ces mêmes régions. Si on se réfère aux conclusions fournies par les modèles, il apparaît que ces domaines spectraux nouvellement étudiés sont bien adaptés à la recherche d'objets protostellaires.

Un objet protostellaire reste néanmoins difficile à observer car c'est un objet de petite dimension qui émet l'essentiel de son rayonnement dans l'infra-rouge où les techniques demeurent encore assez peu sensibles.

Par contre, comme c'est un objet particulièrement dense par rapport au milieu interstellaire, il peut apparaître absorbant en projection sur un champ d'étoiles ou sur une nébuleuse brillante ; c'est pourquoi, historiquement, les premières observations concernant les objets stellaires en formation ont porté sur certaines taches sombres de petite dimension, de forme quasi sphérique et superposées à de grandes régions brillantes. (Globules de Bok.)

La recherche observationnelle dans le domaine des objets protostellaires suit, aujourd'hui, schématiquement trois directions :

1) Mise en évidence d'objets ponctuels ou de faible dimension situés dans des régions où existent déjà des objets reconnus comme jeunes et des accumulations de matière dense : étoiles chaudes, régions ionisées, étoiles à nébulosité. On cherche particulièrement des astres rayonnant dans l'infra-rouge, puisque, selon les modèles, une importante quantité de poussière est contenue dans une enveloppe circumstellaire. La principale difficulté est alors de discriminer ces objets intrinsèquement infra-rouges des étoiles ayant artificiellement un excès infra-rouge dû à l'absorption sélective d'un nuage situé devant l'étoile.

2) Recherche des effets cinématiques de la formation (fragmentation, effondrement, collision de nuages) par des mouvements de matière caractérisés par certains effets sur les profils des raies moléculaires: déplacement, élargissement, self absorption.

3) Déduction de la présence d'étoiles jeunes masquées par le nuage qui les contient, par la modification des conditions physiques du milieu environnant: formation de régions ionisées, illumination de nébuleuses par réflexion, expulsion d'une enveloppe, échauffement des poussières et des molécules autour de l'étoile.

3.2 OBJETS OBSERVÉS DANS LES RÉGIONS DE FORMATION D'ÉTOILES. - 3.2.1 Localisation. - II existe dans les galaxies des régions privilégiées pour la formation stellaire. Les étoiles $\mathrm{O} / \mathrm{B}$ (les plus chaudes et les plus massives) et leurs nébuleuses associées (régions d'hydrogène ionisé appelées région $\mathrm{H}$ II) sont des objets très jeunes (quelques millions d'années). L'examen d'un cliché en $\mathrm{H} \alpha$ (raie de recombinaison la plus intense émise dans les régions ionisées) d'une galaxie extérieure telle que M 51 montre que ces objets ne sont pas distribués au hasard mais tracent à l'intérieur de cette galaxie des bras spiraux. Ce résultat est également confirmé par les observations en continu radio (Mathewson et al., 1972) [16]. Ces bras seraient produits par une onde de densité accompagnée d'une onde de choc dont l'effet serait d'accoître la pression de la matière galactique diffuse. C'est en arrière de ces bras spiraux que les conditions requises pour la formation stellaire semblent réunies.

3.2.2 Nuages sombres denses. - Un des résultats importants des efforts parallèles de la radio-astronomie et de l'infra-rouge lointain $(300 \mu$ à $1 \mathrm{~mm})$ a été la détection et la cartographie de ces objets très massifs $\left(10^{3}\right.$ à $\left.10^{5} \mathrm{M}_{\odot}\right)$ en raies moléculaires de $\mathrm{CO}, \mathrm{H}_{2} \mathrm{CO}$ et $\mathrm{HCN}$, etc., ... (voir pour plus de détails la conférence de F. Combes) et en émission thermique des poussières froides. Ces grands nuages sont aujourd'hui reconnus comme le milieu générateur d'étoiles (proximité de nébuleuses ionisées, par exemple Orion, ou d'amas d'étoiles jeunes).

Les cartes en infra-rouge ou en millimétrique et en raies de $\mathrm{CO}$ sont en général coïncidentes et les pics d'émission $\mathrm{CO}$ sont également des pics d'émission infra-rouge (ce qui tend à prouver que les grains et le gaz sont intimement mêlés). Ces pics ont permis de localiser certaines étoiles invisibles qui chauffent le milieu (Vrba et al., 1975) [17].

3.2.3 Les globules sombres. - Ces objets appelés globules de Bok, du nom de l'astronome qui les étudie depuis plusieurs dizaines d'années, ont des diamètres de l'ordre de quelques dizaines de milliers d'unités astronomiques $\left({ }^{5}\right)$ et des masses de l'ordre de grandeur de la masse du Soleil, ils peuvent absorber jusqu'à dix magnitudes. Jusqu'à présent ils n'ont été détectés que par leur absorption dans le domaine optique et plus récemment dans les raies de $\mathrm{H}_{2} \mathrm{CO}$ (Rickard et al., 1977) [18].

La détection de ces objets dans l'infra-rouge lointain rayonné par le contenu de poussière serait intéressante pour déterminer la température des grains et une valeur précise de la masse. Toutefois les estimations actuelles semblent montrer, qu'en général, ils ne sont pas gravitationnellement liés : ils ne sont donc pas dans une phase de contraction gravitationnelle (Bok et al., 1972) [19].

3.2.4 Les objets ponctuels infra-rouges. - Les objets en formation sont d'autant plus difficiles à mettre en évidence qu'ils sont plus jeunes. La température et les dimensions des objets de faible masse au début de l'effondrement ne sont pas suffisantes pour qu'ils apparaissent en I. R. Dans l'état actuel de la technologie on ne peut espérer observer, dans leurs premières phases d'évolution, que des objets massifs $\left(M \gtrsim 20 \mathrm{M}_{\odot}\right)$. Encore faudra-t-il discriminer les étoiles en formation qui rayonnent directement leur éner-

(5) 1 unité astronomique $=$ distance moyenne Soleil Terre $=1,5 \times 10^{11} \mathrm{~m}$. 
gie en infra-rouge des étoiles déjà formées mais encore enfermées dans un nuage (optiquement épais en visible) qui convertit, par l'intermédiaire des grains de poussière qu'il contient, les rayonnements $\mathrm{U}$. V. et visible en infra-rouge.

L'observation du rayonnement free free radio permet, en principe, de lever l'indétermination: si l'objet est une étoile déjà formée, chaude à sa surface $(25000 \mathrm{~K})$, le rayonnement ultra-violet qu'elle émet ionise une partie de l'enveloppe. Le rayonnement free-free émis, dans le domaine radio, par cette région ionisée, doit alors être observé car il n'est pas absorbé par le nuage neutre qui l'entoure. Par contre, si l'objet central n'est pas suffisamment évolué, sa température est basse et il ne rayonne que dans l'infra-rouge.

Un exemple frappant est celui de la source IRS 5 du grand complexe W 3. Sa luminosité en infra-rouge est équivalente à $10^{5} \mathrm{~L}_{\odot}\left({ }^{6}\right)$. Or, il ne lui correspond aucune source radio (Fig. 3). Au contraire la source IRS 3 est très brillante à la fois en continu radio (région $\mathrm{HII}$ compacte) et en infra-rouge ; elle présente donc les caractéristiques d'une étoile chaude de la

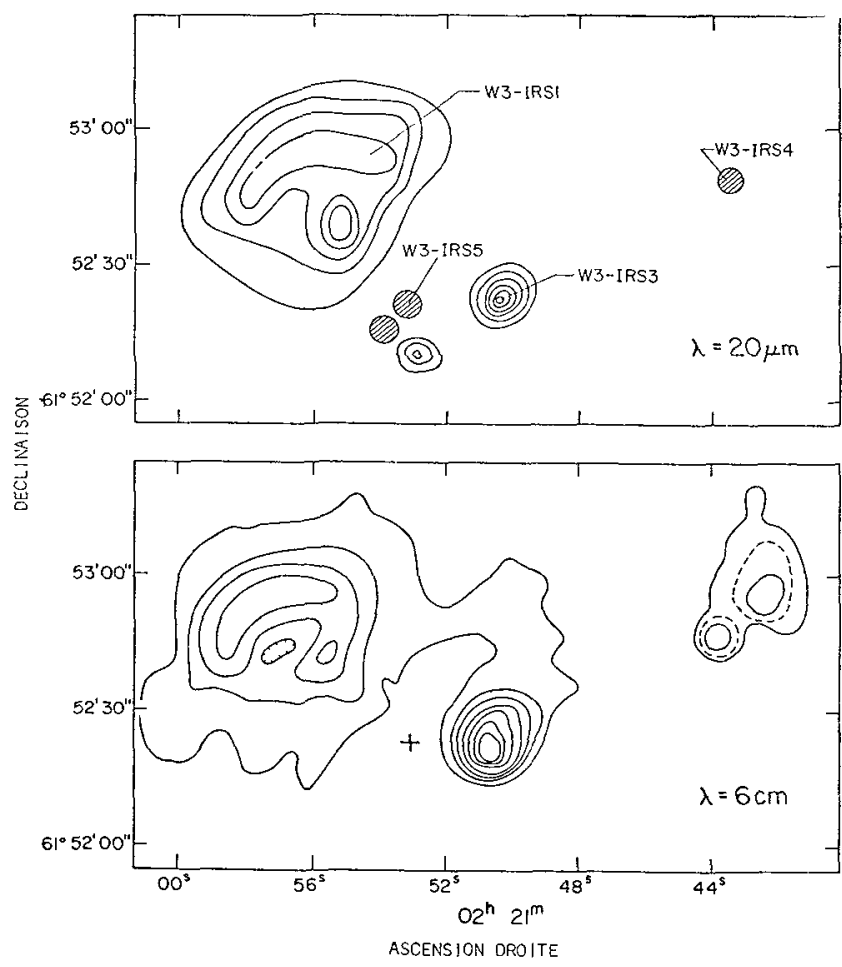

Fíg. 3. - Cartes de la région W 3 en infra-rouge à $20 \mu$ et en continuum radio à $6 \mathrm{~cm}$. Cet ensemble est constitué d'une source étendue qui apparaît en continu radio et en infra-rouge (IRS 1), d'une région H II compacte (IRS 3) qui témoigne de la présence d'une étoile chaude encore enfermée dans le nuage de poussière (rayonnement I. R.) mais capable d'ioniser le gaz, d'une source très lumineuse en infra-rouge (IRS 5) sans contrepartie radio, bon candidat de source protostellaire. La source IRS 4 faible en radio pourrait être dans une situation intermédiaire (région HII en cours d'expansion). (D'après WynnWilliams et al., 1972 [20].)

(๑) $\mathrm{L}_{\odot}=$ luminosité solaire $=4 \times 10^{26} \mathrm{~J} \cdot \mathrm{s}^{-1}$. série principale (voir l'exposé de J. P. Baluteau). Cet exemple montre que des objets dans des états d'évolution très différents peuvent coexister dans le même ensemble.

Le spectre des objets du type IRS 5 se présente en général sous la forme d'un spectre de corps noir à quelques centaines de degrés $K$ avec quelques bandes d'absorption larges attribuées aux silicates (vers $10 \mu$ ) et à la glace (vers $3 \mu$ ) (Wynn Williams et al., 1972) [20] (Fig. 4).

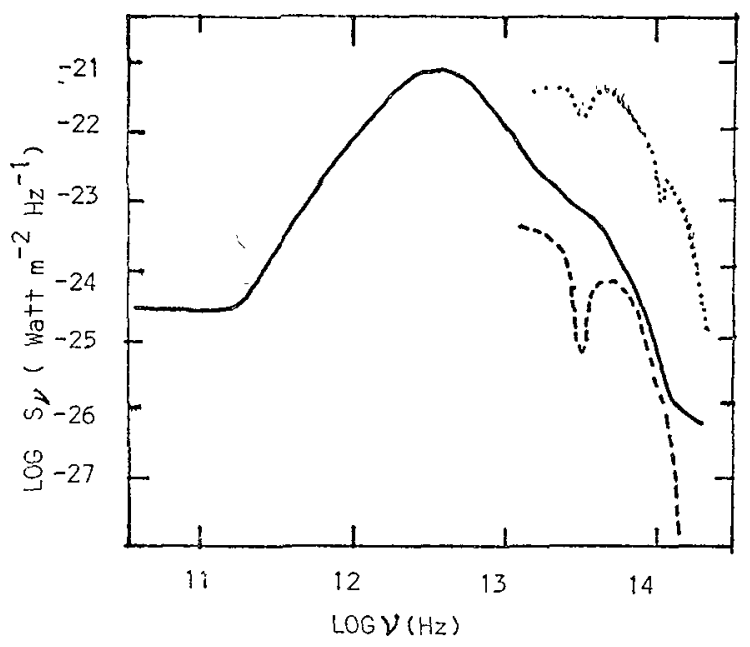

FIG. 4. - Spect re de W 3 (trait plein). L'émission est due à des poussières froides (maximum vers $100 \mu$ ). Spectre de IRS 5 de W 3 (tirets) et de l'objet de Becklin et Neugebauer (BN) de la nébuleuse d'Orion (pointillés, l'échelle des flux est augmentée de 2 unités). On voit apparaître nettement la bande des silicates à $10 \mu$ et, sur l'objet BN, la bande des silicates à $3 \mu$. (D'après Wynn-Williams et al., 1972 [20].)

3.2.5 Les sources maser $\mathrm{OH}$ et $\mathrm{H}_{2} \mathrm{O}$. - De nombreux masers $\mathrm{OH}$ et $\mathrm{H}_{2} \mathrm{O}$ ont été détectés à proximité des régions de formation stellaire. Beaucoup d'incertitudes existent encore à leur sujet : sont-ils des objets protostellaires ou des conséquences de la formation stellaire? Quel est le mécanisme de pompage? Une discussion de la physique de ces objets, est faite dans la conférence de Nguyen Quang Rieu; on se bornera ici à résumer quelques faits observationnels.

Les mesures en synthèse d'ouverture ont permis de montrer que ces sources maser de quelques U. A. d'extension sont associées en groupe d'étendue $10^{3}$ à $10^{5}$ U. A.

Les conditions d'excitation des masers interstellaires sont celles observées dans les régions de formation stellaire : densité de l'ordre de $10^{7}$ à $10^{9} \mathrm{~cm}^{-3}$, températures de l'ordre de 500 à $1000 \mathrm{~K}$ et rayonnement infra-rouge. Les raies d'émission maser $\mathrm{OH}$ et $\mathrm{H}_{2} \mathrm{O}$ sont généralement totalement distinctes. Les sources $\mathrm{OH}$ paraissent associées aux régions $\mathrm{H}$ II compactes tandis que les masers $\mathrm{H}_{2} \mathrm{O}$ sont souvent localisés en bordure de régions $\mathrm{H}$ II et coïncident parfois aux erreurs d'expérience près, avec des sources infrarouges (IRS 5 dans W 3). Dans le cas de W 49 on a 
observé la variation simultanée de l'intensité de plusieurs sources $\mathrm{H}_{2} \mathrm{O}$ distinctes ce qui conduit à penser qu'il ne s'agit pas d'objets individuels mais de taches excitées par la même source, variable, mais non connue

3.2.6 Objets protostellaires dans la phase finale. La phase finale de la formation d'une étoile conduit à l'apparition d'un objet visible optiquement. On dispose depuis longtemps de beaucoup d'informations sur ces objets (Strom et al., 1975) [2] qui présentent les caractères suivants :

- spectre d'émission superposé à un spectre d'étoile normale (chaude pour les étoiles $\mathrm{Ae} / \mathrm{Be}$ de Herbig, plus froide pour les T Tauri),

- profils des raies montrant une éjection (ou, peut-

être dans certains cas, une accrétion) de matière, variabilité irrégulière,

- excès infra-rouge et polarisation optique,

- ils sont le plus souvent en association.

Ces étoiles semblent donc constituées d'un noyau en équilibre hydrostatique : le spectre sous-jacent est celui d'une étoile normale, entourée d'une enveloppe circumstellaire qui se manifeste par un excès infrarouge et une polarisation optique. Cette enveloppe semble en voie de dissipation : les profils de raie peuvent être interprétés en supposant que la matière est soumise à un champ de vitesses radiales dirigé vers l'extérieur ; mais cette interprétation, qui est la plus généralement admise, n'est pas la seule possible. Tous ces caractères s'accordent bien avec les prévisions de la phase finale des modèles. De nombreuses inconnues subsistent encore : on ne s'accorde pas sur les masses de ces objets, sur l'interprétation des profils de raies, sur l'origine de la variabilité. Enfin on a observé, pour certaines $\mathrm{T}$ Tauri (FU Ori et V 1057 Cyg), un brusque accroissement d'éclat après lequel elles deviennent des étoiles normales. Ce phénomène semble marquer le terme de la phase hydrodynamique. On ne sait pas s'il caractérise l'achèvement normal de la phase pré-série principale de toutes les étoiles.

En conclusion une région de formation stellaire est caractérisée par la présence de l'ensemble ou d'une partie des objets suivants :

- Un nuage neutre moléculaire de grande densité $\left(N \sim 10^{6} \mathrm{~mol} / \mathrm{cm}^{3}\right)$, de température $T<100 \mathrm{~K}$ et de masse comprise entre $10^{3}$ et $10^{5} \mathrm{M}_{\odot}$, détectable en raies de $\mathrm{CO}, \mathrm{H}_{2} \mathrm{CO}$ et $\mathrm{HCN}$ (suivant la densité) et en infra-rouge lointain. Les pics de $\mathrm{CO}$ correspondent, en général, aux pics I. R. et souvent à des sources ponctuelles dans l'infra-rouge proche.

- Une ou plusieurs nébuleuses émissives (région H II) produites par le rayonnement d'étoiles très chaudes de grande masse plus ou moins cachées par une enveloppe de poussières. Ces nébuleuses $\left(T \sim 10^{4} \mathrm{~K}\right.$, $n_{\mathrm{e}} \sim 10^{4} \mathrm{~cm}^{-3}, M \sim 50 \mathrm{M}_{\odot}$ ) apparaissent dans différentes raies ioniques, en continu radio ou en $\mathbf{I}$. $R$. moyen. Les isophotes $I$. $R$. et radio coïncident.
- Un amas d'étoiles infra-rouges, invisibles optiquement, de grande luminosité $\left(L \sim 10^{5} \mathrm{~L}_{\odot}\right)$ et situé le plus souvent dans les régions les plus denses des nuages moléculaires ( $\rho$ Oph, NGC 1333).

- Des sources masers $\mathrm{OH}$ et $\mathrm{H}_{2} \mathrm{O}$ associées aux sources infra-rouges.

- Des amas d'étoiles jeunes de type T Tau.

3.3 EXEMPLE DE RÉGION DE FORMATION STELLAIRE : LA NÉBULEUSE D'ORION. - La nébuleuse d'Orion est connue depuis longtemps pour contenir des objets très jeunes : étoiles $\mathrm{O} / \mathrm{B}$, étoiles $\mathrm{T}$ Tau, ... Elle est relativement proche de nous $(500 \mathrm{pc})$.

Cet ensemble comprend la nébuleuse optique bien connue M 42 excitée par un amas d'étoiles de type $\mathrm{O} / \mathrm{B}$ appelé amas du Trapèze.

La cartographie de cette zone en continu radio (Martin et al., 1974) [21] montre que la région ionisée est beaucoup plus étendue que la région visible en optique : une grande partie du rayonnement optique est donc absorbé par la poussière contenue dans le nuage. La masse de cet objet est de $10 \mathrm{M}_{\odot}$ environ, sa densité de $10^{4} \mathrm{~cm}^{-3}$.

Cette région ionisée est associée à un ensemble beaucoup plus vaste comprenant un complexe de molécules et de poussières de température $70 \mathrm{~K}$, de densité $10^{6} \mathrm{~mol} . \mathrm{cm}^{-3}$ et de masse $500 \mathrm{M}_{\odot}$ environ, détecté dans les raies de nombreuses molécules (Harvey et al., 1974) [22].

Une cartographie de l'ensemble de la région dans les raies de $\mathrm{CO}$ en émission montre, en fait, une structure très complexe et très étendue, avec plusieurs pics d'intensité qui permettent de penser que les contrastes de densité - c'est-à-dire une fragmentation - existent. La distance entre les deux principaux pics (OMC 1 et OMC 2) est à peu près de $12^{\prime}$. De plus Kutner et al. (1976) [23] ont observé un gradient de vitesse entre ces deux composantes. Ces raies de $\mathrm{CO}$ ont des profils larges - quelques $\mathrm{km} . \mathrm{s}^{-1}$ - qui ne peuvent être attribués uniquement à l'agitation thermique (une raie émise par un nuage à $100 \mathrm{~K}$ a une largeur thermique de $0,4 \mathrm{~km} . \mathrm{s}^{-1}$ ). Plusieurs hypothèses ont été proposées pour expliquer ces élargissements : microturbulence, ondes hydromagnétiques, petits nuages à grande dispersion de vitesse. L'hypothèse de l'effondrement du nuage semble toutefois la plus solide. L'observation de la self-absorption sur les raies indique que l'on a, sur une même ligne de visée, deux régions de même vitesse donc que la distribution croît vers le centre; une variation de $V(r)$ en $r^{-1 / 2}(r$ : distance au centre du nuage) permet de représenter correctement l'ensemble des profils observés dans les différentes régions du nuage. Mais il faut être prudent car on n'est jamais sûr qu'un seul champ de vitesse soit capable de reproduire les profils observés.

En infra-rouge lointain (Westbrook et al., 1976) [24] à $100 \mu$ et (Werner et al., 1976) [25] à $1 \mathrm{~mm}$ les maxima de l'émission coïncident avec les pics des raies moléculaires. Des amas d'objets ponctuels dans l'infra- 


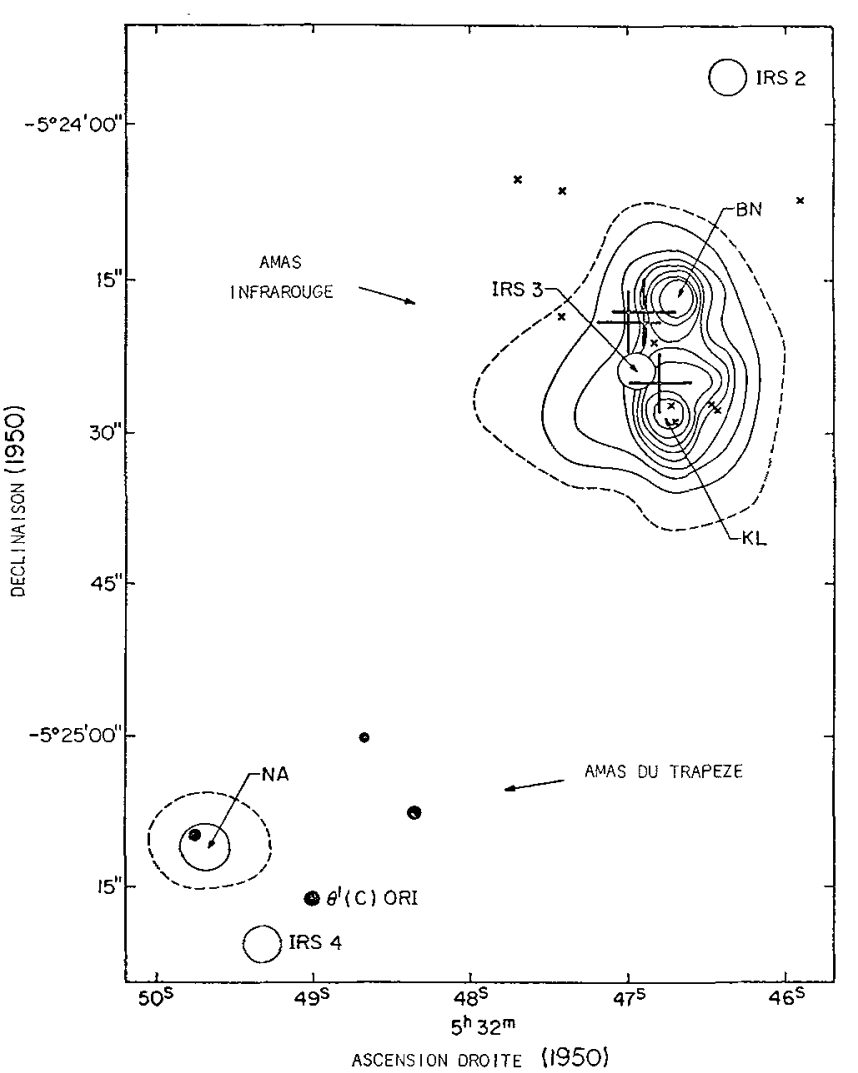

FIG. 5. - Carte de la région centrale du nuage moléculaire d'Orion montrant la répartition spatiale des objets de cet amas d'étoiles en formation. Les isophotes sont celles du rayonnement infra-rouge à $20 \mu$, les cercles représentent les sources ponctuelles infra-rouges, les grandes croix les sources maser $\mathrm{OH}$, les petites croix les sources maser $\mathrm{H}_{2} \mathrm{O}$. (D'après WyNN-WILliams et Becklin, Publ. Astron. Soc. Pac. 86 (1974) 5.) rouge proche (Fig. 5) sont aussi associés spatialement à ces pics. Pour OMC 1, c'est la Nébuleuse de Kleinmann et Low; elle contient l'objet de Becklin et Neugebauer dont la luminosité $\left(10^{4} \mathrm{~L}_{\odot}\right)$ et la température $(600 \mathrm{~K})$ semblent caractéristiques de l'étape d'accrétion prévue par les modèles. L'ensemble de l'amas a une luminosité de plus de $10^{5} \mathrm{~L}_{\odot}$ et n'est visible ni en optique ni dans le continu radio. Son âge est estimé à $5 \times 10^{4}$ ans.

$\mathrm{Au}$ deuxième pic d'intensité des raies- moléculaires (OMC 2) est aussi associé un amas de sources ponctuelles infra-rouges mais de luminosité totale très inférieure.

Des sources maser $\mathrm{OH}$ et $\mathrm{H}_{2} \mathrm{O}$ sont associées à la nébuleuse de Kleinmann et Low et ont fait l'objet d'études détaillées tant pour leur répartition spatiale que leur variabilité temporelle (Baudry et al., 1974 [26] ; Moran et al., 1973 [27]).

A partir de cet ensemble de données on peut construire une représentation spatiale du complexe d'Orion (Zuckermann, 1973 [28]), (Fig. 6), dans laquelle la nébuleuse optique apparaît comme une région ionisée à la surface du nuage moléculaire dont le (ou les) cour(s) plus dense(s) contiennent des étoiles nouvellement formées ou en train de naître.

4. Conclusion générale. - L'échelle de temps de formation d'une étoile, même très massive, est trop grande pour permettre à un observateur de suivre l'évolution d'un objet pré-stellaire. Dans certains cas, on a pu observer des variations notables et, semble-t-il, irréversibles des conditions physiques régnant dans

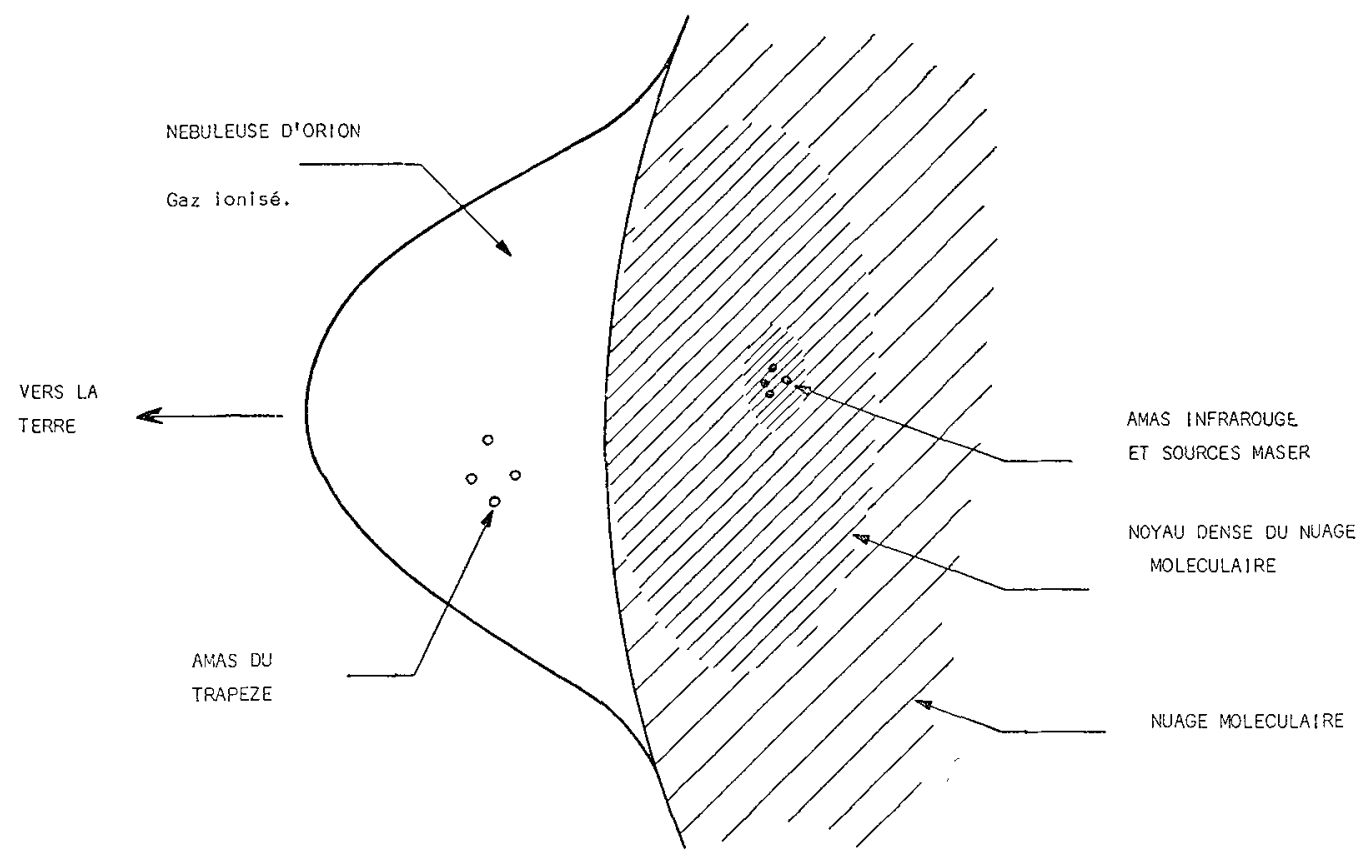

Frg. 6. - Modèle spatial de la nébuleuse d'Orion déduit des mesures I. R. optique et radio.

(Daptès ZuCKERMANN, 1973 [28].) 


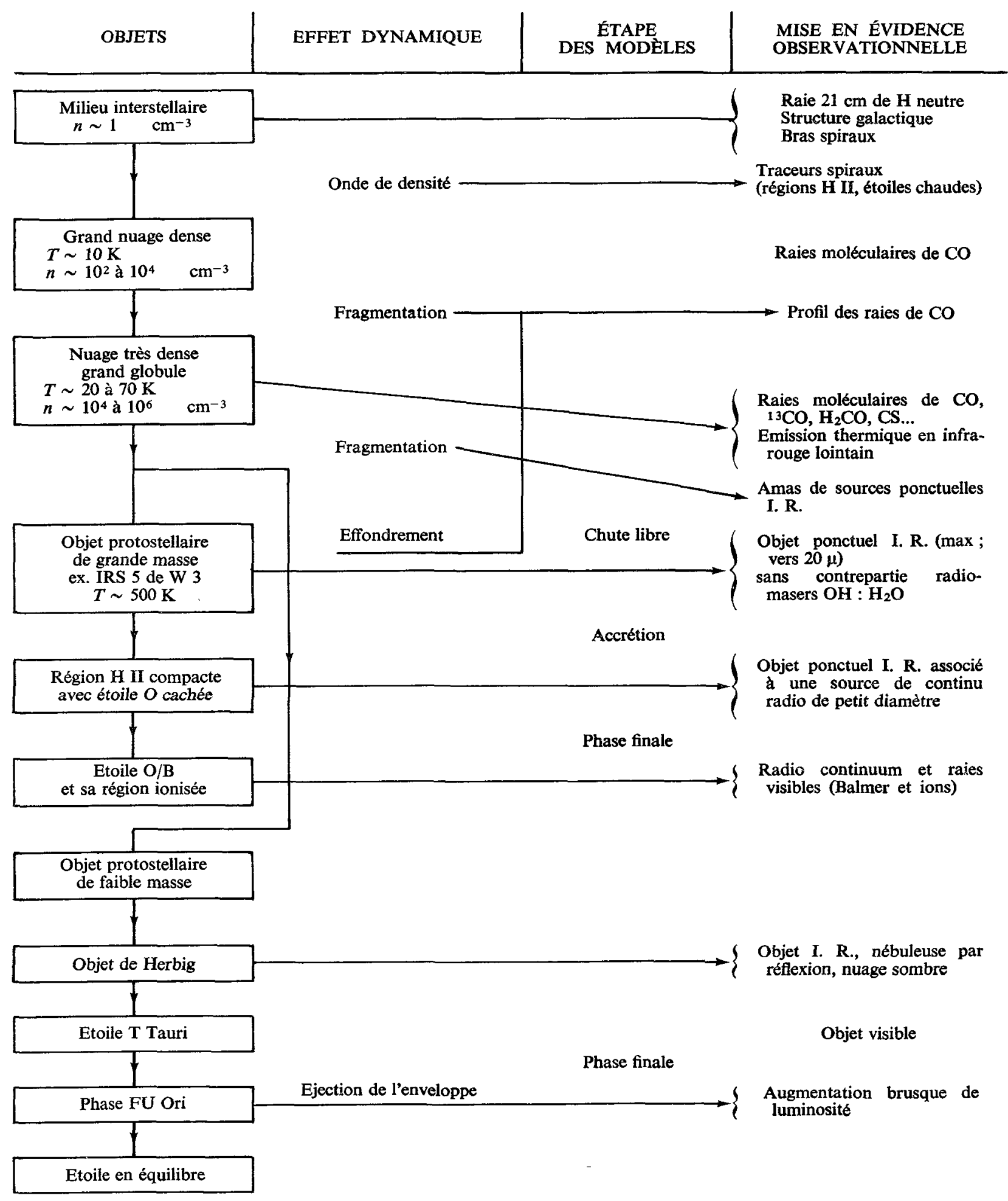

FIG. 7. -- Une séquence évolutive phénoménologique. 
un objet: c'est le cas de l'accroissement brusque de luminosité de certains objets très jeunes (FU Ori, V 1057 Cyg) suivi d'une transformation radicale de la nature de l'étoile, de la modification de la'structure spatiale de certaines nébulosités. Ces événements restent néanmoins exceptionnels et, bien que d'un grand intérêt dans la compréhension de la physique des objets jeunes, on doit dire, qu'en apportant subitement une abondance d'informations, ils soulèvent plus de questions qu'ils n'éclairent les problèmes déjà ouverts. On ne doit donc pas espérer observer directement les métamorphoses d'un objet et l'on devra s'accomoder du puzzle de données fournies par des objets différents, de masses différentes, dans des environnements différents et dans des états d'évolution différents, puzzle dont on devra induire une séquence évolutive pour un objet donné. Le schéma de la figure 7 propose une séquence évolutive phénoménologique, telle qu'elle peut être imaginée à partir des observations actuellement disponibles; évidemment elle présente encore beaucoup d'incertitudes.

S'il peut être satisfaisant pour l'esprit de chercher à faire coïncider les faits observationnels avec les prédictions théoriques des modèles, cette manière de voir paraît dangereuse car elle a tendance à rigidifier les interprétations et à censurer certaines hypothèses surtout dans un domaine aussi neuf que celui-ci.

Si la confrontation entre théorie et observation a été poussée très loin dans certains domaines comme la structure et l'évolution des étoiles, où une quantité considérable de données a été accumulée depuis plus de 100 ans, elle n'est pas possible avec précision dans le domaine de la formation des étoiles.

En effet, il y a à peine 15 ans que l'on commence a mesurer des paramètres (en dehors de l'absorption des globules de Bok) qui ont un rapport avec la formation stellaire; et ces mesures sont relativement pauvres en information : résolution spatiale insuffisante en raies moléculaires et en infra-rouge, sensibilité insuffisante des mesures en infra-rouge qui nécessitent beaucoup des temps d'observation pour recueillir peu d'information.

De plus, la construction des modèles de protoétoiles fait intervenir beaucoup plus de phénomènes physiques difficiles à représenter et de paramètres libres que celle de modèles d'étoiles déjà formées.

Lorsque seront disponibles les nouveaux instruments : télescopes optiques modernes de grand diamètre (télescope de $3,60 \mathrm{~m}$ de l'E. S. O. au Chili, télescope de 3,60 m Canada-France-Hawaï), le Grand Interféromètre Millimétrique (destiné à obtenir des cartes radio des nuages moléculaires avec une résolution comparable à l'optique et à mettre en évidence des objets de petit diamètre encore plus jeunes que ceux détectés en infra-rouge), les projets d'interférométrie visible et infra-rouge, les projets d'expériences spatiales en infra-rouge (navette spatiale), les détecteurs infrarouge en mosaïque, etc., ..., (pour ne citer que des instruments à participation Française ou Européenne), les résultats observationnels seront affinés et interagiront efficacement avec les modèles théoriques qui, on l'espère, disposeront de moyens puissants.

\section{Bibliographie}

[1] Mc Nally, D., Rep. Prog. Phys. 34 part 1 (1971) 71.

[2] Strom, S. E., Strom, K. M. et Grasdalen, G. L., Ann. Rev. Astron. Astrophys. 13 (1975) 187.

[3] Stein, R. F., MC Cray, R. et Schwarz, J., Astrophys. $J$. Lett. 177 (1972) L 125.

[4] Parker, E. B., I. A. U. Symposium no 39 (1970) 168.

[5] Wielen, R., Publ. Astron. Soc. of the Pacific 86 (1974) 341.

[6] Appenzeller, I. et Tscharnuter, W., Astron. Astrophys. 30 (1974) 423.

[7] Reddish, V. C., Mon. Not. R. Astron. Soc. 170 (1975) 261.

[8] Richtmyer, D. et Morton, K. W., Difference Methods for initial-value Problems (Interscience Publishers New York, London, Sydney 2nd edition) 1967, 295.

[9] KolesniK, I. G., Astrometriya Astrofiz. 28 (1976) 79.

[10] Larson, R. B., Ann. Rev. Astron. Astrophys. 8 (1973) 219.

[11] Westbrook, C. K. et TARTer, C. B., Astrophys. J. 200 (1975) 48.

[12] Narita, S., Nakano, T. et Hayashi, C., Prog. Theor. Phys. 43 (1970) 942.

[13] Bertout, C., Astron. Astrophys. 51 (1976) 101.

[14] Yorke, H. W. et KRÜGel, E., Astron. Astrophys. 54 (1977) 183.

[15] AppenZeller, I. et Wolf, B., Astron. Astrophys. 54 (1977) 713.

[16] Mathewson, D. S., Van den Kruit, P. C., Brouw, W. N., Astron. Astrophys. 44 (1972) 73.

[17] Vrba, F. J., Strom, K. M., Strom, S. E., Grasdalen, G. L., Astrophys. J. 197 (1975) 77.
[18] Ruckard, L. J., Palmer, P., Buhl, D., Zuckerman, B., Astrophys. J. 213 (1977) 654.

[19] Bok, B. J., Cordwell, C. S. and Cromwell, R. H. Dark Nebulae, Globules and Protostars (The University of Arizona Press) 1971.

[20] Wynn-Williams, C. G., Becklin, E. E., Neugebauer, G., Mon. Not. R. Astron. Soc. 160 (1972) 1.

[21] Martin, A. H. M., Gull, S. F., Mon. Not. Astron. Astrophys. 32 (1974) 231.

[22] Harvey, P. M., Gatley, I., Werner, M. W., Elias, J. H., Evans, N. J., Zuckerman, B., Morris, G., Sato, T., LitvaK, M., Astrophys. J. Lett. 189 (1974) L 87.

[23] Kutner, M. L., Evans II, N. J., Tuckner, K. D., Astrophys. J. 209 (1976) 452.

[24] Westbrook, W. E., Werner, M. W., Elias, J. H., Gezari, D. Y., Hoser, M. G., Lo, K. Y., Neugebauer, G., Astrophys. J. 209 (1976) 94.

[25] Werner, M. W., Gatley, I., Harper, D. A., Becklin, E. E., Loewenstein, C., Telesco, C. M., Thronson, H. A.. Astrophys. J. 204 (1976) 420.

[26] Baudry, A., Forster, J. R., Welch, W. J., Astron. Astrophys. 36 (1974) 217.

[27] Moran, J. M., Papadopoulos, G. D., Burke, B. F., Lo, K. Y., Schwartz, P. R., ThaCKNER, D. L., Johnston, K. J., KNowles, S. H., Reisz, A. C. and Shapiro, I. I., Astrophys. J. 185 (1973) 535.

[28] ZuckermanN, B., Astrophys. J. 183 (1973) 863. 